\title{
APUNTE LINGÜÍSTICO-FILOSÓFICO
}

\author{
STEFANO ARDUINI \\ (Conservatorio de Venecia)
}

Para las breves notas que quiero desarrollar en estas páginas es posible obtener una indicación de un artículo de Julius Moravcsik publicado en The Behavioral and Brain Sciences (1980, $n .^{\circ} 3$ ) y en traducción italiana en apéndice a Regole e Rappresentazioni de N.A. Chomsky. Aun siendo esenciales, las observaciones de Moravcsik son muy claras y precisas. En primer lugar propone la distinción entre teorías profundas, que se refieren a niveles de hechos inobservables en sus explicaciones, y teorías superficiales, que, adhiriéndose to más posible al observable, postulan inobservables sólo si ello es inevitable. Las primeras son, pues, las comúnmente aceptadas por la ciencia ${ }^{\text {; }}$; las segundas se han mostrado, en cambio, incapaces de adecuarse a ciencias como la física moderna. Es, sin embargo, una curiosa anomalía histórica que, cuando tenemos que tratar de ciencias sociales, éstas demuestran una singular predilección por las teorías superficiales de la mente, con una indudable resistencia a referirse a hechos inobservables, "quanto meno per quanto riguarda meccanismi complessi che

1 Las teorias profundas "sono guidate dall'intuizione secondo cui le apparenze osservabili possono essere spiegate adeguatamente solo dall'esame di aspetti sottostanti e non osservabili della natura" (Moravcsik, 1981: 233). Siendo ésta la esencia de la ciencia moderna, es probable entonces que tenga razón Paul Feyerabend cuando afirma que la ciencia está mucho más cercana al mito de lo que una filosofía científica está dispuesta a admitir (Feyerabend, 1979). 
obbediscano a principi astratti.» (Moravcsik, 1981: 234). Es, en cambio, bien diversa la posición de Chomsky, quien ha optado por una teoría profunda:

La sua argomentazione si basa suil'analogia tra le teorie della fisica e di altre scienze di successo e quello che si dovrebbe tentare di realizzare nelle scienze sociali. Se le teorie 'profonde' sono state quelle che hanno ottenuto successo nelle scienze naturali, perché non ci si dovrebbe aspettare che la stessa cosa valga per le scienze sociali? (Chomsky, 1981: 234) ${ }^{2}$.

El porqué de esta situación es dilucidado por Moravcsik en múltiples factores históricos y es en las resistencias a las teorías profundas donde es hallada la causa de la oposición a la concepción modular de la mente, es decir, a la hipótesis "dell'esistenza di strutture diverse alla base di competenze come la padronanza del linguaggio, la capacità di ragionare, e la capacità di calcolo" (Chomsky, 1981: 236). Chomsky ha basado esta elección, según Moravcsik, en una analogía, precisamente en la analogía con las ciencias biológicas en las que son postuladas estructuras diversas para las distintas funciones vitales. Este argumento nos interesa de modo particular también porque es indudable que uno de los puntos más importantes de la reciente gramática generativa atañe precisamente a la discusión en torno a la llamada concepción modular de la mente (Chomsky, 1975; 1977; 1980; 1981; 1982). Tal concepción nos conduce además a varias consideraciones y nos lleva a formular diversas hipótesis incluso si actualmente las pruebas empíricas a favor son escasas y débiles. Pero tal vez no nos encontramos demasiado desventajados respecto al ansia continua de la gramática generativa de producir pruebas, visto que, a nuestro parecer, este ansia

2 Observaciones de este tipo van acompañadas en general (también en Chomsky) por afirmaciones acerca del estilo de Galileo y de su validez para el método científico. Es una pena que Chomsky invoque como testimonio de cientificidad, en las ciencias sociales, a autores quizás hoy un poco desacreditados: «Non vi e ragione di abbandonare l'approccio generale delle scienze naturali quando ci si rivolge allo studio degli esseri umani e della societa. Ogni serio approccio a simili argomenti cerchera, con maggiore o minor successo, di adottare lo 'stile galileiano'. L'economia politica marxista, con le sue astrazioni di vasta portata, ne è un chiaro esemplo: infatti, 'qui si tratta delle persone soltando in quanto sono la personificazione di categorie economiche, incarnazione di determinati rapporti e di determinati interessi di classi' (Marx, 1867) e il capitale è considerato non 'una cosa, bensl un determinato rapporto di produzione sociale, appartenente ad una determinata formazione storica della societa. Rapporto che si presenta in una cosa e da a questa cosa un carattere sociale specifico' (Marx, 1865, trad. it.: 1095-1096); e in generale, l'economia e considerata in ultima analisi come uno studio dei rapporti di classe" (Chomsky, 1979: 353). 
se traduce en buenos propósitos más que en acción real. Esto naturalmente no es acusación y quizás a este propósito tiene razón Feyerabend cuando escribe:

Secondo i nostri risultati attuali, difficilmente una teoria può mai essere in accordo con i fatti. Anche la richiesta di ammettere solo quelle teorie che siano in accordo con i fatti disponibili e accettati ci lascerebbe senza alcuna teoria. (Feyerabend, 1979: 55)

Según la concepción modular la mente está organizada en un conjunto de sistemas cognitivos que están estructurados en modos relativamente autónomos. Una hipótesis de este género condiciona naturalmente incluso las posibles interpretaciones sobre lo que es una teoría del aprendizaje.

¿Cómo debería proceder un científico al estudiar el problema? Elegiría un organismo y un dominio cognitivo determinado e intentaría construir la teoría del aprendizaje de aquel organismo en aquel dominio.

Esta teoría - llamémosla TA $(O, D)$ - puede concebirse como un sistema de principios, un mecanismo, una función, que tiene una determinada "entrada" y una determinada "salida" (su dominio y su alcance, respectivamente). La "entrada» del sistema TA (O, D) será un análisis que de los datos de $\mathrm{D}$ haga $\mathrm{O}$; la "salida» (que es, evidentemente, una representación interna, no patente y manifiesta) será una estructura cognitiva de un determinado tipo. Esta estructura cognitiva es un elemento del estudio cognitivo alcanzado por O. (Chomsky, 1975, trad. esp., 1979: 27).

Ahora, imaginando haber aislado un cierto número de $\mathrm{TA}(\mathrm{O}, \mathrm{D})$, podemos intentar algunas respuestas alternativas a la pregunta sobre lo que es una teoria del aprendizaje. En particular: 1) en cualquier caso en que elegimos $O$ y $D$, encontramos la misma $T A(O, D)$; 2) existen rasgos significativos comunes a todas las $T A(O, D)$; 3 ) existe un conjunto de dominios $D_{1} \ldots, D_{n}$ tal que las varias TA sean iguales o presenten semejanzas interesantes. Chomsky da una respuesta negativa al primer punto; considérense a título de ejemplo los seres humanos de una parte y las ratas de otra, considérense además el dominio del lenguaje y el del recorrido de un laberinto. Suponiendo que 1) fuese verdadera, deberiamos tener la misma mayor habilidad de los seres humanos respecto a las ratas tanto en recorrer un laberinto como en aprender el lenguaje. Esto, naturalmente, no es verdadero y confirma la di- 
versidad en que están estructurados los sistemas cognitivos de la mente humana ${ }^{3}$.

En cuanto a la pregunta 2), si bien más plausible, no permite en el estado actual de los conocimientos una respuesta adecuada. Con respecto al punto 3) Chomsky sostiene que "debería existir alguna forma de delimitar un conjunto de dominios que permitan una respuesta positiva a 3). Si así fuera, podríamos decir que dentro de estos límites el organismo aprende de una forma similar o idéntica en diversos dominios cognitivos" (Chomsky, 1975, trad. esp., 1979: 36).

Si éste es el cuadro dentro del cual se ofrece la hipótesis modular de la mente, podemos en cualquier caso poner como punto firme el hecho de que ai principi di acquisizione delie strutture cognitive non sono solo diversi da specie a specie ma anche specifici di ogni dominio cognitivo", además, "dire che la mente è modulare significa dire che consiste di sistemi separati, ognuno con la sua propria struttura, ognuno designato specificamente per trattare un particolare tipo di prob/emi; tutto il sistema poi interagirebbe in modo di creare un sistema molto complesso di strutture altamente specializzate" (Scalise, 1980:

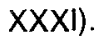

Establecemos ahora mediante hipótesis una TA particular, la de los hombres en el dominio de la verdad científica. Si quisiéramos intentar ser más precisos deberíamos hablar, sin embargo, de varios dominios de la verdad científica. Está claro que no tenemos ninguna prueba empírica de que esto sea verdad; podría ser perfectamente que el dominio sea uno solo y que por tanto debamos concentrar nuestros esfuerzos sobre una sola TA. En el estado actual de nuestros conocimientos empíricos estas dos posiciones con aceptables. Existen, no obstante, varias pruebas indirectas de que esta interpretación sea la exacta: por ejemplo, el hecho de que históricamente la ciencia haya estado en realidad subdividida en diversos dominios. Debe ser algo más que un mero accidente histórico el hecho de que la historia de la ciencia haya procedido según una cada vez más marcada identificación

3 Esto está relacionado con otro problema: se podría suponer que la distinción TA (H, $L$ y $T A(R, R L)$ (con $H=$ hombres; $L=$ lenguaje; $R=$ ratas; $R L=$ recorrer un laberinto) sea extensible también a los monos y a otros animales sin que esto implique una escala de valores, es decir, que sea un puro accidente de la evolución que los hombres se hayan encontrado disponiendo de una TA $(H, L)$ mejor que las de las otras especies de animales. Si esto hace inútiles los intentos de enseñar el lenguaje a otras especies animaies no implica sin embargo una superioridad, si no histórico-cultural, de la especie humana sobre otras que pueden ser superiores en otros dominios; por ejemplo, para la pareja $T A(H, L)$ y TA $(R, R L)$ ciertas ratas blancas son sin duda más hábiles que los hombres en este tipo de aprendizaje. 
de dominios particulares y que esto no haya sido dificultado por los varios intentos de unidad de la ciencia desde Llull a Neurath. Se debería reflexionar, además, sobre un hecho muy curioso e importante: que la ciencia moderna haya llegado a resultados notables exactamente en el momento en que ha ido especificando cada vez más los dominios particulares. La ciencia antigua ha llegado frecuentemente a intuiciones muy próximas a las que han llevado a los descubrimientos modernos, pero si ha perdido con frecuencia es precisamente porque, confundiendo los dominios, ha intentado aplicar teorías no adecuadas a aquéllos.

Pensando apropiadamente se podría hipotizar que todo esto deba tener que ver con las capacidades de la mente humana, con su estructura. Imaginamos, pues, volviendo a lo arriba expuesto, una mente modular, esto es, una mente constituida por varios sistemas separados, cada uno designado para tratar un tipo particular de problemas, y que este tipo de problemas no sean otra cosa que los distintos dominios de las ciencias históricas o, mejor, de las que se han manifestado en la realidad como las diversas ciencias históricas, definidas por consiguiente como los dominios específicos de la mente humana. Coherentemente con las afirrnaciones precedentes habrá por tanto unas TA de ciertos dominios científicos que no serán las mismas TA de otros dominios científicos. Es perfectamente lícito suponerlo, así como la TA del lenguaje no es la misma que la TA de recorrer laberintos. No solamente se puede suponer además que en ciertas TA de un dominio particular somos más hábiles que en otras, propiamente como somos más hábiles en la TA ( $H, L)$ que en la TA $(H, R L)$. Podria incluso ser, consiguientemente, que el dominio del estudio del lenguaje no sea el mismo del de otros fenómenos físicos y que las dos TA sean por ello diversas. No existen pruebas empíricas de esto, pero podría ser una hipótesis, una hipótesis que explicaria, sin embargo, ciertas dificultades epistemológicas en el estudio del lenguaje: por ejemplo la dificultad de estudiar el lenguaje como un fenómeno físico o históricamente las dificultades, las resistencias de los diferentes estudiosos de la materia a querer considerar a los seres humanos como simples organismos biológicos en el mundo natural: y ésta sería una explicación mucho más aceptable que la esclerosis que habría golpeado el mundo académico en su totalidad.

Chomsky compara el "órgano mental" lenguaje humano a otros órganos: el corazón, etc.; no nos son dadas pruebas de que efectivamente sea así, o mejor, no tenemos pruebas que no partan de la fe de que sea así; todo lo más se trata de pruebas del tipo de las aportadas para sostener la idea clásica contraria por Boas, Sapir, etc. No nos interesa cancelar el paralelismo lingüístico que podemos tranquila- 
mente conservar, pero por la debilidad de las pruebas podríamos imaginar, como ha imaginado, por lo demás, una buena parte de la tradición, que los dos órganos sean diferentes, incluso podríamos pensar que la tradición en realidad no ha inventado nada si se la halla constreñida a adoptar esta distinción en cuanto la mente humana ha debido hacer cuentas con la diversidad de los dominios y las dificultades en que tropiezan las reunificaciones. Se podría suponer, además, que las teorias científicas diversas y distinciones del tipo físico-mental ${ }^{4}$ sean en realidad el espejo de los diversos modos que la mente ha adoptado (que ha sido constreñida a adoptar) para afrontar los varios dominios a los que poderse aplicar.

Incluso podriamos no saber, ni estar nunca en grado de saber, por qué se trata de dominios diversos, sino simplemente rendirnos al hecho de que nuestra mente afronta estos dominios como diversos.

(Agradezco a Tomás Albaladejo su traducción al español).

\section{Referencias bibliográficas}

Chomsky, N. A. (1975), Reflection on Language, New York, Pantheon Books (trad. esp. Reflexiones sobre el lenguaje, Barcelona, Ariel, 1979).

- (1977), Essays on Form and Interpretation, Amsterdam, North Holland (trad. esp. Ensayos sobre forma e interpretación, Madrid, Cátedra, 1982).

- (1978), "Una teoria della grammatica centrale», en: Rivista di grammatica generativa, III, pp. 3-30.

- (1979), «Linguaggio», en: Enciclopedia Einaudi, VIII, pp. 352-399.

- (1980), Rules and Representations, New York, Columbia University

Press (trad. esp. Reglas y representaciones, México, F.C.E., 1983).

- (1981), Lectures on Government and Binding, Dordrecht, Foris.

4 Con ello no pretendo avalar una concepción dualista dei saber. Considero más bien que la ciencia moderna sea un conjunto de modelos diversamente interrelacionados (propiamente como, por lo demás, las varias TA interactúan entre sí) y que el conjunto del saber sea una red con tantos modos distintos pero obviamente ligados a los otros (las varias TA son propiamente distintas pero inevitablemente interrelacionadas). Esta verja se desarrolla en sentido horizontal (sincrónico) o vertical (histórico): tarea del científico es la de añadir nuevos nudos o la de conseguir recorrer esta doble red buscando en ella una cierta racionalidad. 
- (1982), Some Concepts and Consequences of the Theory of Government and Binding, Cambridge, MIT Press.

Feyerabend, P. K. (1979), Contro il metodo, Milano, Feltrinelli.

Graffi, G. (1980), "L'evoluzione del programma di ricerca di $N$. Chomsky", introducción a N. A. Chomsky, Forma e interpretazione, Milano, II Saggiatore, 1980, pp. 8-62 (trad. it de Essays on Form and Interpretation).

Moravcsik, J. (1981), "La radicale rottura di Chomsky nei confronti della tradizione moderna», epílogo a N. A. Chomsky, Regole e reppresentazioni, Milano, II Saggiatore, 1981, pp. 233-237 (trad. it. de Rules and Representations).

Scalise, S. (1980), "Dalla teoria standard alla teoria standard estesa", introducción a N. A. Chomsky, Riflessioni sul linguaggio, Torino, Einaudi, 1980, pp. VII-XLV (trad. it. de Reflection on Language). 